\title{
Sonographic Detection of Placenta Accreta in The Second and Third Trimesters of Pregnancy
}

Ahmed Mohammad Hamada ${ }^{1, *}$ MSc., Mofeed Fawzy Mohammad ${ }^{2}$ MD., Ahmed Mohammad El Sadek ${ }^{2}$ MD.

* Corresponding Author:

Ahmed Mohammad Hamada

hamada_mido141414@yahoo.com

Received for publication August 02, 2021; Accepted September 17, 2021; Published online September 17, 2021.

Copyright The Authors published by Al-Azhar University, Faculty of Medicine, Cairo, Egypt. Users have the right to read, download, copy, distribute, print, search, or link to the full texts of articles under the following conditions: Creative Commons Attribution-Share Alike 4.0 International Public License (CC $B Y-S A$ 4.0).

doi: 10.21608/aimj.2021.87673.1535

${ }^{I}$ Department of Obstetrics \& Gynecology, Kafr El-Sheikh General Hospital, Egypt.

${ }^{2}$ Department of Obstetrics \& Gynecology, Faculty of Medicine, AlAzhar University, Cairo, Egypt.

\section{ABSTRACT}

Background: the term morbid adherent placenta suggests a nontypical placental implantation into the uterine barrier and was utilized to express placental accreta, percreta, and increta. Placental accreta (PA) is a placental where the placenta villi stick on straightly to the myometrium. Objective: this work aim to evaluate the specificity, sensitivity, and diagnosis accurateness of ultrasound (US) in placental accretes diagnosing in the 2nd and 3rd trimesters of gestation.

Patients and Methods: This was a prospective research that was performed at obstetrics and gynecology departments of both El Hussein and Kafr El-Sheikh Hospitals during the period between January 2020 till July 2020 aiming for evaluating the accurateness of US in the placenta accreta diagnosing.

Results: The US sensitivity was (100\%), specificity was (85 \%), NPV was $(100 \%)$ and PPV was $(90.9 \%)$ with accuracy of $(94 \%)$ in placental accreta diagnosing.

Conclusion: Ultrasonography has satisfactory sensitivity for placental accreta diagnosing; but specificity doesn't seem to be good as concluded in other reports. US still the most sensitive and frequently utilized scanning modality for the placental accreta diagnosing as it is precise, cheap, and noninvasive and save time. This modality has excellent popularity as it is extensively accessible, patients friendly, and comparatively cheap.

Keywords: Ultrasound; Placenta accretes; Prenatal diagnosis; Pregnancy, Sensitivity.

Disclosure: The authors have no financial interest to declare in relation to the content of this article. The Article Processing Charge was paid for by the authors.

Authorship: All authors have a substantial contribution to the article.

\section{INTRODUCTION}

Placental accreta (PA) happens when the placenta partially or wholly attacked and inseperable from the uterine barrier. 3 levels of anomalous placenta attachments are described in accordance to the attack depth: accreta (chorionic villi attachrd to the myometrium), increta (chorionic villi attack into the myometrium), percreta (chorionic villi attack to the myometrium and serosa, and infrequently into near organs, like the bladder). ${ }^{1}$

The PA incidence has raised and seems to be analogous to the elevated rate of cesarean deliveries. Early uterine surgical operation, myomectomies and curettages, as well as cesarean sections, have all been accompanying with anomalous placentation, but further ominously, placenta previa was accompanied with an elevated risk of PA. PA happened in $9.3 \%$ of cases with placental previa and in $0.004 \%$ of females with no placental previa. ${ }^{2}$

Surprising conditions of PA may cause catastrophic bleeding, many complications like adult respiratory distress syndrome (RDS), renal failures, Sheehan's syndrome (SS), and even mortality. The profit of performing the PA diagnosis earlier to birth is that it permits for multi-disciplinary arranging in a try to minimalize possible mothers or newborns morbidities and mortalities. ${ }^{3}$

The diagnostics are frequently made using US and the characteristics expressive of PA comprise placental vascular cavities, myometrium thinning covering the placenta, retro-placental losing "clear cavity", placental protrusion to the bladder, raised vascularity of the uterine serous membrane and turbulent blood flowing via the lacunae on DopplerUS. $^{4}$

US is the principal way for diagnosing the placental attack; however, when it is essential to describe the lesion topography in connection to pelvic soft tissue, when there are unclear US results or a doubt of a backward PA, with or with no placental previa, US can be inadequate and placenta magnetic resonance imaging (MRI) is needed. ${ }^{2}$

Total, gray-scale US looks to be a brilliant means for diagnosing PA in females at risk. Its sensitivity 
ranging between $(77-87 \%)$, with a specificity between (96 - $98 \%$ ), a positive predictive value (PPV) of (65-93\%), and a negative predictive value (NPV) of (98\%). A metanalysis in 2013 concluded a joint sensitivity of $83 \%{ }^{5}$

On the other hand, one more new research proposed that the accurate predictive power of US in PA diagnosing is really lesser than formerly stated. The authors reported that primary researches evaluating the US prediction accurateness of PA can be influenced by adding a single expert notes, recognized suspicions for accreta, and knowing the risk-factors. Furthermore, small sample size, reviewing strategies, and extensive changeability in the definitions and inclusion criteria result in contradiction in performances and skewed sensitivities. ${ }^{6}$

When researchers are blinded to clinical histories and multi qualified providers reviews a similar US scans, the PA diagnosing accuracy drops. The sensitivity, specificity, PPV and NPV, and accuracy drop to (54, $88,82,65 \& 65 \%$ ), respectively, in accordance to the largest prospective research of accreta cases up to the present time. ${ }^{7}$ The true sensitivity and specificity expected to lie anywhere amid practitioners are unusually know about cases history and risk-factors and may utilize instantaneous sonographic scanning and $3 \mathrm{D}$ methods. ${ }^{6}$

Limited researches matching the 2 ways were performed and all are small and deficient of statistic power. MRI can be complementary to US in patients where the placenta is backward or placed sideways. ${ }^{8}$ It is significant to identify that when an MRI was utilized in combination with US in evaluating cases for the PA, MRI was infrequently beneficial in altering operative treatment. One exclusion can present in MRI's capability to measure the degree/depth of placental attack and discriminating placenta percreta from accretes. ${ }^{9}$

\section{PATIENTS AND METHODS}

This was a prospective investigation that was conducted at obstetrics and gynecology departments of both El Hussein and Kafr El-Sheikh Hospitals during the period between January 2020 till July 2020 aiming to evaluate the accurateness of US in the PA diagnosing

A total of 100 cases $(n=100)$ had been gathered from the outpatient clinics of both hospitals who will be diagnosed by US to have PA.

Primary outcome: Evaluation of sensitivity, specificity and diagnosing accurateness of US in PA diagnosing

Secondary outcomes: Assessment of the effects of placenta accretes on the maternal and fetal outcomes. Inclusion criteria: All cases that had been suspected to have placenta accrete by US during the work period. Patient approval will be obtained before the procedure.
Exclusion criteria: Presence of medical disorders (diabetes, hypertension, epilepsy, etc.), primigravida cases, twin pregnancy, patient refuse and presence of fetal anomalies

All patients will be subjected to:

I- History taking

Personal history: Names - ages -occupations parities - singular behaviors of medical significance) and Family history, menstrual history: current pregnancy ages determining in accordance to last catamenial period and confirmation by ultrasound assessment. Present history: Presence of uterine contraction. Associated symptoms: (bleeding, abdominal pain). Obstetric history: for each previous delivery: Number, ante-partum periods (preceding pre-term labour or miscarriage), post-partum and purperium periods and previous history of miscarriage. Surgical history: Any cervical surgeries, previous cesarean section.

II- Examination General Examination: Vital signs (Blood pressure (BP), temperature, pulse, breathing rates; body mass index (BMI). and blood pressure), Chest \& heart examination and Limbs examinations. Obstetric examination: Fundal level \& fundal grip.

III- Ultrasound examination: US with linear and sectorial 3.5- \& 5-MHz trans-ducers and as well 5- \& $7-\mathrm{MHz}$ trans-vaginal trans-ducers will be used at both obstetrics and gynecology departments of ElHussein and Kafr El-Sheikh General Hospital to detect findings considered as criteria of PA.

On grayscale US scanning, we will the existence of at minimum one of the next features to specify PA (involving its variants, placental increta and placental percreta): full losing of the retro-placental sonolucent region, Asymmetrical retro-placental sonolucent region. Tinning or disruptions of the hyperechoic uterine serosal-bladder boundary, Existence of focally exophytic masses attacking the urine bladder, Existence of anomalous placenta spaces. They frequently look to be linear, parallel, vascular canals spreading from the placenta parenchyma to the myometrium, these objects differs from vascular ponds in that they look more unclear and display disordered flow, while ponds seem more rounds with laminal flowing. Decreased myometrial thickness $(<1$ $\mathrm{mm})^{10}$.

Similarly, the PA diagnosing was considered as positive when any one of these color Doppler ultrasound (CDUS) standards was existing: Diffused or focally lacunar flowing patterns Sonolucent vascular ponds with disordered flowing typifed by high-speed (peak systolic speed $>150 \mathrm{~mm} / \mathrm{sec}$ ) and small-resistance wave-form, Hyper-vascularity of the uterine-bladder interfaces with anomalous blood vessels connecting the bladder to the placenta (zones of raised vascularity and continuum of lacunar flow from the placenta through the myometrial layer without interfering clear spaces), Obviously expanded vessels over the bordering sub-placental area ${ }^{10}$.

Ultrasound results will be retrospectively investigated and matched with operative results. Furthermore, the placenta penetrating degree and its 
definite topography will be confirmed in the surgical room clinically and anatomically.

Statistical Analysis: Data were examined if normally distributed via the Shapiro Walk testing. Qualitative data have been presented as frequency and percentage. Chi square testing $(\chi 2)$ and Fisher exact has been utilized to determine differences among qualitative data as showed. Quantitative data have been presented as mean \pm SD (Standard deviation).

\section{RESULTS}

Patients were between the ages of 24 and 56 with mean age of $33.42 \pm 9.21$-yrs, meanwhile mean BMI is $26.86 \pm 3.75 \mathrm{~kg} / \mathrm{m}^{2}$. Number of previous CS ranged from $2-4$. Table (1)

\begin{tabular}{|c|c|}
\hline & $\begin{array}{l}\text { Patients } \\
(\mathrm{n}=100)\end{array}$ \\
\hline $\begin{array}{l}\text { Age (years) } \\
\text { Mean } \pm \text { SD } \\
\text { Range }\end{array}$ & $\begin{array}{l}33.42 \pm 9.21 \\
24-56\end{array}$ \\
\hline $\begin{array}{l}\text { BMI }\left(\mathrm{kg} / \mathrm{m}^{2}\right) \\
\text { Mean } \pm \mathrm{SD} \\
\text { Range }\end{array}$ & $\begin{array}{l}26.86 \pm 3.75 \\
22-32\end{array}$ \\
\hline $\begin{array}{l}\text { No. of previous CS } \\
\text { Mean } \pm \text { SD } \\
\text { Range }\end{array}$ & $\begin{array}{l}2.93 \pm 0.873 \\
2-4\end{array}$ \\
\hline
\end{tabular}

Table 1: Demographical features of the contributed cases.

$66 \%$ of the patients were diagnosed as PA by ultrasonography. Table (2)

\begin{tabular}{||l|c|c||}
\hline \multirow{2}{*}{} & \multicolumn{2}{|c|}{ Patients $(\mathrm{n}=100)$} \\
\cline { 2 - 3 } & $\mathrm{N}$ & $\%$ \\
\hline Placenta accrete & 66 & 66 \\
\hline Non Accreta & 34 & 34 \\
\hline
\end{tabular}

Table 2: Ultrasound findings among the patients

Of 66 accreta in US findings, 60 patients were accreta by surgical observation with false positive was $15 \%$. Table (3)

\begin{tabular}{|l|l|l|l|l|l|l|}
\hline \multirow{3}{*}{ US } & \multicolumn{3}{|l|}{ Surgical findings } & \multirow{3}{*}{ Total } & \multirow{2}{*}{$\mathrm{P}$} \\
\cline { 2 - 5 } & Accreta & \multicolumn{2}{|l|}{ Non-acceta } & \\
\cline { 2 - 5 } & $\mathrm{N}$ & $\%$ & $\mathrm{~N}$ & $\%$ & & \\
\hline Accreta & 60 & $100 \%$ & 6 & $15 \%$ & $66(66 \%)$ & \\
\hline Non-accreta & 0 & -- & 34 & $85 \%$ & $34(34 \%)$ & \multirow{2}{*}{$<0.001$} \\
\hline Total & 60 & $100 \%$ & 40 & $100 \%$ & & \\
\hline
\end{tabular}

Table 3: Comparison of US findings according to surgical findings

The US specificity was ( $85 \%)$, sensitivity was (100 $\%)$, NPV was (100\%) and PPV was (90.9\%) with accuracy of $(94 \%)$ in PA diagnosing. Table (4)

\begin{tabular}{||l|l|l|}
\hline Statistic & Value & $95 \%$ CI \\
\hline Sensitivity & $100 \%$ & $88.43 \%-100 \%$ \\
\hline Specificity & $85 \%$ & $62.11 \%-96.79 \%$ \\
\hline Positive Predictive Value (PPV) & $90.91 \%$ & $77.89 \%-96.59 \%$ \\
\hline Negative Predictive Value (NPV) & $100 \%$ & -- \\
\hline Accuracy & $94 \%$ & $83.45 \%-98.75 \%$ \\
\hline
\end{tabular}

Table 4: Diagnostic value of US

\section{DISCUSSION}

The term morbid adherent placenta suggests a nontypical placental implantation into the uterine barrier and was utilized to label placental accreta, percreta, and increta. PA is a placental where the placenta villi stick on straightly to the myometrium. Placental increta happens when the placental villi enter into the myometrium and placental percreta happens when the villi attack via the myometrium and into serosal. PA is a main cause of mother morbidities and mortalities and is presently the main cause for peripartum hysterectomy. ${ }^{11}$

The exact reason isn't definite, but it was assumed to be connected to the injuries of the decidua basalis, which permits for the placenta attacks into the myometrium. The wall function of the decidua is lost in these conditions, and the invasive trophoblasts can enter the myometrium to changing lowest point, from the shallowest PA to deeper myometrial attack PA, with breaches of the uterine serous membrane and can assault adjacent organs. ${ }^{12}$

Risk-factors for PA comprise preceding cesarean deliveries, placental previa mother ages $>35$-yrs, uterine instrumentations and intra-uterine scar, all of which can be linked with injury to or deficiency of the decidual basalis. Within the incidence of these risk-factors, the accoucheurs have an elevated index of uncertainty for PA and take appropriate protections. Exactly, this situation should be combined into diagnosing differentially in cases with preceding caesarean births and frontal low-lying placenta. ${ }^{13}$

Many studies have proposed that neither colorDoppler nor power-Doppler could measure uteroplacental vascularizing or add any further data to grayscale US scanning in PA diagnosing. But several literatures on this topic either define single patients or deficiencies suitable control (i.e., placental previa with no accreta), hardening the validation of the present criteria. ${ }^{14}$

The current work aimed to evaluate the sensitivity, specificity, and diagnostical accurateness of US in the diagnosis of placental accretes in the $2^{\text {nd }} \& 3^{\text {rd }}$ gestational trimesters.

This was a prospective investigation that was conducted at on a total of 100-pregnancies at obstetrics and gynecology departments of both El Hussein and Kafr El-Sheikh Hospitals during the period from January 2020 till July 2020.

Analysis of our findings, Patients were between the ages of 22 and 60 with mean age of $32.31 \pm 8.44$-yrs, meanwhile mean BMI is $26.92 \pm 3.65 \mathrm{~kg} / \mathrm{m}^{2}$. Number of previous CS ranged from $2-4$.

In agreement with our findings, the study of Khodair et al., ${ }^{15}$ included 50 pregnant women aged 23-38-yrs with a mean age $31.18 \pm 3.88$. All of the included subjects had previous cesarean section scar, 17 out of 50 had single previous scar, 19 had two previous 
scars, 11 had three previous scars and 3 had six previous scars.

Ornaghi et al., ${ }^{16}$ had ages median of 35-yrs (IQR, 31.4-39.0-yrs) at births; 6-cases were $>45$-yrs and single case $<20$-yrs. There were $74.5 \%$ births via CS, with elective surgical operation was the commones (73.4\%). Total, $54 \%$ had a preceding CS, with $18.5 \%$ and $6.5 \%$ having 2 or $\geq 3$ preceding CS, respectively.

Borg et al., ${ }^{11}$ reported that the ages mean of the involved cases was $31.24 \pm 3.86$-yrs and ranging from 23 to 38 -yrs.

Furthermore, the present study reported that the mean parity of patients was $3.16 \pm 1.204$, and mean gravidity was $4.08 \pm 1.46$.

Borg et al., ${ }^{11}$ reported that their gravidity ranging from 2 to 11 with the mean was $4.0 \pm 2.06$ also their parities ranging from 1 to 4 with parities mean was $1.96 \pm 0.789$. in regard to gestation ages, it was ranging from 31 to 39 -wks with a mean value 35.88 \pm 2.14 -wks.

Moreover, Hamisaa et al., ${ }^{17}$ reported that the ages mean of this work group was 29-yrs ranged between 20 and 40-yrs. Between cases with approved PA, the ages mean was 32.25 -yrs ( 25 to 40 -yrs). The parities mean of cases ranging between 1 and 6 with a mean value of 1.6. The mean CS-number between the studied group was 1.6 ranged between $1 \& 5$. The mean CS- number between cases approved with PA: 2.75 ranging between $2 \& 5$.

In the present study, we found that $60 \%$ (30 patients) had PA intraoperatively.

In agreement with our findings, the study of Borg et al., ${ }^{11}$ reported that intraoperatively $(64 \%)$ of patients were PA while, $(36 \%)$ of them were non-PA.

El Wakeel et al., ${ }^{10}$ reported that a number of 20pregnants with placenta previa have been statistically evaluated in the present work, with ages mean of 30.96-yrs. Their gestation age ranging between 28 to 37 weeks, eleven of these 20 patients had a PA diagnosed clinically at birth, pathologically, or both. Their ages ranging between $20 \& 37$-yrs with ages mean of 29.6-yrs, and all of them had undergone preceding uterine operations. Among the 11 cases confirmed with PA, 3-cases have placenta percreta, 6-cases have PA, and 2-cases ahve placenta increta.

In the current study, we found that $66 \%$ of the patients were diagnosed as PA by ultrasonography.

In a study done by Shweel et al., ${ }^{18}$, regarding the CDUS results 15/28 (53.5 \%) cases were PA positive and 13/28 (46.4\%) were negative.

Lopes et al., ${ }^{19}$ reported that between the 37-cases (100\%) who experienced the US, the PA- prevalence was $20(54.2 \%)(95 \%$ confidence interval $[95 \% \mathrm{CI}]$ : 40.8-67.3).
On the other hand, in the present study, 33 accreta in US findings, 30 patients were accreta by surgical observation with false positive was $15 \%$.

In accordance with our findings, Shweel et al., ${ }^{18}$, surgically and pathologically our 28 comprised cases approved the PA diagnosing in 11/28 (39.2\%) cases. CDUS was correct in 10/15 (66.6\%) cases, 5-cases incorrect positive and 1-case incorrect negative.

Imaging plays a crucial role in the PA diagnosing. CDUS was the initial diagnostical device for placental evaluation. The anomaly scan done at 18-20-wks of pregnancy provides a perfect chance for disease screening. Placenta previa, placental lacunae, anomalous CDUS imaging patterns, deficiency of the retro placental clear spaces, and decreased myometrial width were detected in the PA diagnosing. An asymmetrical bladder barrier proposes the opportunity for placenta percreta. The existence of lacunae has the maximum sensitivity allowing identification of accreta in $78-93 \%$ of cases. $^{20}$

Interestingly, in the present study, US sensitivity of $(100 \%)$, specificity (85\%), NPV $(100 \%)$ and PPV (90.9\%) with accurateness of $94 \%$ in PA diagnosing

In comparison with the study of Borg et al., ${ }^{11}$ concluded that sensitivity for the PA diagnosing was $(100 \%)$ for CDUS, $(93.7 \%)$ for 2DUS and (75\%) for MRI. Specificity was (66.6\%) with CDUS and $(77.7 \%)$ for 2DUS and $(55.5 \%)$ for MRI. The uppermost PPV was revealed in 2DUS (88\%), MRI had the lowermost PPV (75\%).

In a systematic review of Pagani et al., ${ }^{21}$ in which 7 researches involving 721-cases at risk for AIP investigated the diagnostical performances of US in the detection of the placenta attack severity built on histo-pathology. US has a total good diagnosing accurateness in recognizing the depth of placenta attack with sensitivities of $90.6 \%$ (95\% CI 80.796.5), $93.0 \%$ (95 \% CI 80.9-98.5), 89.5\% (95\% CI 73.2-96.3), and 81.2\% (95\% CI 51.8 - 94.6) for PA, increta, accrete / increta, and percreta, respectively; the corresponding figures for specificity were $97.1 \%$ (95\% CI 95.4-98.3), 98.4 (95 \% CI 97.0-99.2), 94.7 (95\% CI 91.0-96.9), and 98.9 (95\% CI 95.0-100).

In reviewing Shweel et al., ${ }^{18}$ data CDUS revealed sensitivity $90 \%$, accurateness $78 \%$, specificity $70 \%$, PPV (67\%), and NPV (92\%) for the PA diagnosing. Analogous to our work, Bonnie et al., ${ }^{22}$ concluded that US exhibited (93\% sensitivity; and $71 \%$ specificity). Levine et al., ${ }^{23}$ revealed CDUS has sensitivity $(86 \%)$ and a specificity of $(92 \%)$.

Warshak et al., ${ }^{24}$ concluded that US had sensitivity $(77 \%)$ and specificity (96\%). This inconsistency in sensitivity and specificity in the later report and ours can be clarified on the basis that our findings revealed 5-cases incorrect positive patients, and single case was negative. The low incorrect negative findings in that report can be participated to the fact that all placentas were frontal in location. 
Moreover, in the study of Dwyer et al., ${ }^{14}$ US had sensitivity of (93\%) (95\% CI, 80-100\%) and specificity of (71\%) (95\% CI, 49-93\%)

Lopes et al., ${ }^{19}$ revealed that the sensitivity of the US was $87.5 \%$, with a PPV of $(65.1 \%)$, and a NPV $(75.0 \%)$.

Berkley et al., ${ }^{25}$ reported that US is a brilliant instrument for the PA diagnosing in cases with risk for this irregularity. Its sensitivity was concluded to be ranged from (77-87\%) with specificity of (96-98 $\%)$, a PPV of (65-93\%), and a NPV of (98\%).

Warshak et al., ${ }^{24}$ matched US and post-contrast MRI performances in the diagnosing and assessment of PA. They concluded on 39 patients of approved PA with an un-paired investigation design. US has a sensitivity (77\%) and specificity (96\%), and MRI with gadolinium improvement had a sensitivity (88 $\%)$ and specificity $(100 \%)$. The high statistically values in the afore-mentioned report can be for the reason that they had accomplished routine transvaginal US as well as the trans-abdominal method in evaluating their involved patients in addition to gadolinium enhancement in their MRI test, in accordance to them it had clearer explained the outer placenta superficial comparative to the myometrium. $^{26}$

In conclusion, the PA prevalence is growing, and physicians must be knowledgeable of this entity and its scanning characteristics. US still the greatest sensitive and commonest utilized scanning method for the PA diagnosing as it is precise, cheap, and noninvasive and fast. This way has enjoyed great fame as it is extensively obtainable, case friendly, and comparatively cheap.

\section{CONCLUSION}

Ultrasonography has good sensitivity for PA diagnosing; whereas, specificity doesn't seem to be as good as described in other reports. US still the greatest sensitive and commonest utilized scanning method for the PA diagnosing as it is precise, cheap, and noninvasive and fast. This way has enjoyed great fame as it is extensively obtainable, case friendly, and comparatively cheap.

\section{REFERENCES}

1. Silver, RM. and Branch, DW. Placenta accreta spectrum. New England Journal of Medicine 2018; 378(16), 1529-36.

2. Nunes C, Carvalho RM, Araújo C, et al. Placental accreta diagnosing by ultrasonography: a" gold standard"? Diagnóstico de placenta accreta por ecografia:"gold standard"? Acta Obstet Ginecol Port. 2014; 8(2), 136-40.

3. Morgan EA, Sidebottom A, Vacquier M, et al. The effect of placental location in cases of placenta accreta spectrum. American journal of obstetrics and gynecology, 2019.
4. Jauniaux E, Collins S. and Burton G. J. Placenta accreta spectrum: pathophysiology and evidence-based anatomy for prenatal ultrasound imaging. American journal of obstetrics and gynecology. 2018; 218(1), 75-87.

5. Meng X, Xie L. and Song W. Comparing the diagnostic value of ultrasound and magnetic resonance imaging for placenta accreta: a systematic review and meta-analysis. Ultrasound in medicine \& biology. 2013; 39(11), 1958-65.

6. Berkley E. M. and Abuhamad A. Ultrasound Diagnosis of the Morbidly Adherent Placenta Placenta Accreta Syndrome 2017; 39-52.

7. Bowman ZS, Eller AG, Kennedy AM, et al. Interobserver variability of sonography for prediction of placenta accreta. Journal of Ultrasound in Medicine. 2014; 33(12), 21532158.

8. Rezk MAA, and Shawky M. Grey-scale and colour Doppler ultrasound versus magnetic resonance imaging for the prenatal placental accreta diagnosing. The Journal of MaternalFetal \& Neonatal Medicine 2016; 29(2), 21823.

9. D'antonio F, Iacovella C, Palacios-Jaraquemada $\mathrm{J}$, et al. Prenatal identification of invasive placentation using magnetic resonance imaging: systematic review and meta-analysis. Ultrasound in Obstetrics \& Gynecology 2014; 44(1), 8-16.

10. El Wakeel A, Azab S, and Rahman HMA. Role of ultrasound, Doppler, and MRI in the placental accreta diagnosing. Menoufia Medical Journal 2018; 31(3), 1023.

11. Borg H, Ahmed M, Ossman HA, et al. Alarabawya, Color Doppler ultrasound in placental accreta diagnosing, 2018.

12. Fitzpatrick KE, Sellers $S$, Spark $P$, et al. Incidence and risk factors for placenta accreta/increta /percreta in the UK: a national case-control study. 2012; PLOS ONE 7: e52893.

13. Jang DG, Lee GSR, Yoon JH, et al. Placenta percreta-induced uterine rupture diagnosed by laparoscopy in the first trimester: case report. Int J Med Sci 2011; 8: 424-7.

14. Dwyer BK, Belogolovkin V, Tran L, et al. Prenatal placental accreta diagnosing: sonography or magnetic resonance imaging? Ultrasound Med 2008; 27:1275-81. 
15. Khodair S, Elsokary A and Elabdien Mz, Antepartum Evaluation of Placenta Accreta in Women with Placenta Previa by Color Doppler, Power Doppler Ultrasound and MRI: A Prospective Study, Med. J. Cairo Univ 2020; 88(2): 505-12.

16. Ornaghi S, Maraschini A, Donati S, Regional Obstetric Surveillance System Working Group. Characteristics and outcomes of pregnant women with placenta accreta spectrum in Italy: A prospective population-based cohort study. PloS one 2021; 16(6), e0252654.

17. Hamisaa M, Mashalya E, Samer F, et al, Role of Doppler US and MRI in placental accreta diagnosing, Alexandria Journal of Medicine, Volume 51, Issue 3, ; 225-30

18. Shweel M, Nadia F.El Ameen, Mohamed A.Ibrahiem, Ahmed Kotib, Placenta accreta in women with prior uterine surgical operation: Diagnostic accuracy of Doppler ultrasonography and MRI, The Egyptian Journal of Radiology and Nuclear Medicine 2012; 43: 3: 473-80

19. Lopes ES, Feitosa FEL, Brazil AV, et al Assessment of Sensitivity and Specificity of Ultrasound and Magnetic Resonance Imaging in the Placental accreta diagnosing. Rev Bras Ginecol Obstet 2019; 41(1):17-23.

20. Satija B, Kumar S, Wadhwa L, et al. Utility of ultrasound and magnetic resonance imaging in prenatal placental accreta diagnosing: A prospective study. Indian $J$ Radiol Imaging 2015; 25:464-70.
21. Pagani G, Cali G, Acharya G, et al. Diagnostic accuracy of ultrasound in detecting the severity of abnormally invasive placentation: a systematic review and meta-analysis. Acta Obstet Gynecol Scand 2018; 97: 25-37.

22. Bonnie B, Victoria T, Lan R, et al. Prenatal placental accreta diagnosing: sonography or magnetic resonance imaging? J Ultrasound Med 2008; 27: 275-1281.

23. Levine CA, Hulka J, Ludmir W, et al, Placenta accreta: evaluation with color Doppler US, power Doppler US, and MR imaging, Radiology 1997; 205: 773-7.

24. Warshak CR, Eskander $\mathrm{R}$ and Hull AD. Accuracy of ultrasonography and magnetic resonance imaging in the placental accreta diagnosing. Obstet Gynecol 2006; 108: 573-81.

25. Berkley EM and Abuhamad AZ, Prenatal Placental accreta diagnosing, Is Sonography All We Need?, https://doi.org/10.7863/ultra.32.8.1345, 2013.

26. Lax A, Prince MR, Mennitt KW, et al. The value of specific MRI features in the evaluation of suspected placental invasion. Magn Reson Imaging 2007; 25: 87-93. 